\title{
EFFECT OF SECOND SOUND ON THE ONSET OF RAYLEIGH-BENARD CONVECTION IN A COLEMAN - NOLL FLUID
}

\author{
S. Pranesh*
}

\begin{abstract}
This paper deals with linear stability analysis of the effects resulting from the substitution of the classical Fourier law by the non-classical Maxwell - Cattaneo low in Royleigh - Benard convection in second order fluid is studies. Colemon-Noll constitutive equaion is used to give a viscoelastic correction. The eigenvalue is obtained for free - free isothernal boundary combination. The classical approach predicts an infinite speed for the propagation of heat. The present non-classical theory involves a wave type heat transport (SECOND SOUND) and does not suffer from the physically unacceptable drawback of infinite heat propagation speed. It is found that the results are noteworthy af short times and the critical eigenvalues are less than the classical ones.
\end{abstract}

\section{Introduction}

Viscoelastic fluids can also be modeled by the Coleman-Noll constitutive equation and when this constitutive equation is considered the viscoelastic fluids are termed

* Post Graduate Department of Mathematics, Christ University, Hosur Road, Bangalore. 560 029, INDIA. Phone No.: 080-40129336,E-moil: pronesh.s@christuniversity.in 
as second-order fluids. Second-order fluids are dilute polymeric solutions (e.g., poly-ios-butylene in Cetane, Methyl-Methacrylate in n-butyl acetate, polyethylene oxide in water, etc.). These fluids express stress in terms of a second degree polynomial in rate of strain. Coleman and Noll[1] have studied, for the first time a simple fluid having fading memory and suggested a second order correction to incompressible perfect fluids. Coleman and Noll [1] derived the following constitutive equation for an incompressible second-order fluid;

$$
\tilde{\tau}=-p I+\mu_{1} \tilde{A}_{(1)}+\mu_{2} \tilde{A}_{(2)}+\mu_{3} \tilde{A}_{(1)}^{2}
$$

Where $\vec{\tau}$ is the stress tensor, $\mathrm{p}$ is an indeterminate pressure, $\mu_{1}, \mu_{2}$ and $\mu_{3}$ are measurable material constants and they denote respectively viscosity, elasticity and cross viscosity. These materials can be determined from viscometric flows for any real fluid. $A_{(1)}$ and $A_{(2)}$ are Rivlin-Ericken tensors and they denote respectively the rate of strain and acceleration. Kaloni [2] has made some useful remarks on the parameters and provided useful theorems for second order fluids. Sharma and Kumar [3] has studied the effect of rotation on thermal instability of Rivilin-Ericksen elastic-viscous fluid and they found that rotation has a stablilizing effect and the presence of rotation introduces oscillatory modes and also they obtained the sufficient condition for the non-existence of overstability. Siddheshwar and Srikrishna [4] have siudied linear and non-linear convection in second-order fluid. They found that in the linear theory the critical eigenvalue is independent of viscoelastic effects and principle of exchange of stability holds. An autonomous system of differential equations representing cellular convection arising in the non-linear study is solved numerically. The reported works on convection are with classical Fourier heat flux law.

The drawback of the classical law motivated Lindsay and Stranghan [5], Stranghan and Franchi [6], Lebon and Cloot [7] and Siddheshwar [8, 9] to adopt a nonclassical heat flux law in studying Rayleigh - Benard / Marangoni convection. Pranesh [10] studied the linear stability analysis of the effects resulting from the substitution of the classical Fourier law by the non-classical Maxwell - Cattaneo law in Rayleigh - Benard convection in micropolar fluid. Siddheshwar and Pranesh [1 1] studied the effects of modulation on the onset of convection in an BoussinesqStokes fluid with Maxwell-Cattaneo law in Rayleigh Beanrd convection. The hyperbolic heat equation (SECOND SOUND) model adopted by these authors does not suffer from the physically unacceptable drawback of infinite heat propagation speed. The principle of exchange of stability is also not guaranteed in these problems. 
The objective of this paper is to replace the classical parabolic heat equations by non-classical Maxwell-Cattaneo heat flux law and study Rayleigh-Benard convection in second-order fluids.

\section{Mathematical Formulation}

Consider a horizontal layer of infinite extent occupied by a Boussinesquian, Coleman - Noll fluid of depth $d$. Let $\Delta T$ be the temperature difference between lower and upper flat fluid surfaces. The governing equations for the Rayleigh-Benard situation in a Boussinesquian Coleman - Noll fluid are

$\nabla \cdot \vec{q}=0$

$\rho_{\circ}\left[\frac{\partial \overrightarrow{\mathrm{q}}}{\partial \mathrm{t}}+(\overrightarrow{\mathrm{q}} \cdot \nabla) \overrightarrow{\mathrm{q}}\right]=-\nabla \mathrm{p}-\rho \mathrm{g} \hat{\mathrm{k}}+\nabla \vec{\tau}$

$\tilde{\tau}=-p I+\mu_{1} \tilde{A}_{(1)}+\mu_{2} \tilde{A}_{(2)}+\mu_{3} \tilde{A}_{(1)}^{2}$

$\frac{\partial \mathrm{T}}{\partial \mathrm{t}}+(\overrightarrow{\mathrm{q}} \cdot \nabla) \mathrm{T}=-\nabla \cdot \overrightarrow{\mathrm{Q}}$

$\tau\left[\dot{\bar{Q}}+\vec{\omega}_{1} \times \vec{Q}\right]=-\vec{Q}-\kappa \nabla T$

$\rho=\rho_{0}\left[1-\alpha\left(T-T_{0}\right)\right]$

where

$$
\tilde{A}_{(1)}=\nabla \vec{q}+(\nabla \vec{q})^{T}
$$

and

$\tilde{A}_{(2)}=\dot{\tilde{A}}_{(1)}+\tilde{A}_{(1)}\left[\nabla \overrightarrow{\mathrm{q}}+(\nabla \overrightarrow{\mathrm{q}})^{\mathrm{T}}\right]$ 
The basic state of the fluid being quiescent is described by

$$
\vec{q}_{b}=0, \quad p=p_{b}(z), \rho=\rho_{b}(z), T=T_{b}(z), \vec{Q}_{b}=\left(0,0,-\kappa \frac{\Delta T}{d}\right) .
$$

\section{Linear Stability Analysis}

Let the basic state be disturbed by an infinitesimal thermal perturbation. We now have

$$
\left.\begin{array}{ll}
\overrightarrow{\mathrm{q}}=\overrightarrow{\mathrm{q}}_{\mathrm{b}}+\overrightarrow{\mathrm{q}}^{\prime}, & \vec{\omega}=\vec{\omega}_{\mathrm{b}}+\vec{\omega}^{\prime}, \quad \mathrm{p}=\mathrm{p}_{\mathrm{b}}+\mathrm{p}^{\prime}, \\
\rho=\rho_{\mathrm{b}}+\rho^{\prime}, & T=\mathrm{T}_{\mathrm{b}}+\mathrm{T}^{\prime}, \quad \overrightarrow{\mathrm{Q}}=\overrightarrow{\mathrm{Q}}_{\mathrm{b}}+\overrightarrow{\mathrm{Q}}^{\prime}
\end{array}\right\}
$$

The primes indicate that the quantities are infinitesimal perturbations and subscript $b$ indicates basic state value.

Substituting equation (8) into equations (1) to (7) and non-dimensionalising the linearised equations using

$$
\left(x^{*}, y^{*}, z^{*}\right)=\frac{(x, y, z)}{d}, \vec{q}^{*}=\frac{\vec{q}^{\prime}}{\chi / d}, \vec{\omega}^{*}=\frac{\vec{\omega}^{\prime}}{\chi / d^{2}}, t^{*}=\frac{t}{d^{2} / \chi}, T^{*}=\frac{T^{\prime}}{\Delta T}
$$

we get

$$
\begin{aligned}
& \frac{1}{\operatorname{Pr}} \frac{\partial}{\partial \mathrm{t}}\left(\nabla^{2} \mathrm{~W}\right)=\left(1+\frac{\mathrm{Q}_{\mathrm{s}}}{\operatorname{Pr}} \frac{\partial}{\partial \mathrm{c}}\right) \nabla^{4} \mathrm{~W}+\mathrm{R} \nabla_{1}^{2} \mathrm{~T} \\
& (1+2 \mathrm{C} \sigma) \sigma \mathrm{T}=(1+2 \mathrm{C} \sigma) \mathrm{W}+\nabla^{2} \mathrm{~T}-\mathrm{C} \nabla^{2} \mathrm{~W}
\end{aligned}
$$

where the asterisks have been dropped for simplicity and the non-dimensional parameters $P r, Q_{s}, R$ and $C$ are as defined as,

$$
\operatorname{Pr}=\frac{\zeta+\eta}{\chi}
$$

(Prandtl number), 
$Q_{s}=\frac{\mu_{2}}{\rho_{\mathrm{o}} \mathrm{d}^{2}}$

(Viscoelastic parameter),

$\mathrm{R}=\frac{\alpha \mathrm{g} \Delta \mathrm{Td}^{3} \rho_{0}}{(\zeta+\eta) \chi}$

(Rayleigh number) and

$C=\frac{\tau \chi}{2 d^{2}}$

(Cattaneo number).

Equations (9) and (10) are solved for velocity stress free, isothermal boundaries and hence the assumed boundary conditions are

$\mathrm{W}=\frac{\partial^{2} \mathrm{~W}}{\partial \mathrm{z}^{2}}=\mathrm{T}=0$ at $\mathrm{z}=0, \mathrm{l}$.

The infinitesimal perturbations $W$ and $T$ are assumed to be periodic waves and hence these permit a normal mode solution in the form

$$
\left[\begin{array}{l}
\mathrm{W} \\
\mathrm{T}
\end{array}\right]=\left[\begin{array}{l}
\mathrm{W}(\mathrm{z}) \\
\mathrm{T}(\mathrm{z})
\end{array}\right] \exp [\sigma \mathrm{t}+\mathrm{i}(\mathrm{lx}+\mathrm{my})]
$$

where $\mathrm{l}$ and $\mathrm{m}$ are horizontal components of the wave number $\overrightarrow{\mathrm{a}}$

Substituting equation (12) into equations (9) and (10), we get

$$
\begin{aligned}
& {\left[\frac{\sigma}{\operatorname{Pr}}-\left(D^{2}-a^{2}\right)-\frac{Q_{s} \sigma}{\operatorname{Pr}}\left(D^{2}-a^{2}\right)\right]\left(D^{2}-a^{2}\right) W=-R a^{2} T,} \\
& (1+2 C \sigma) \sigma T=(1+2 C \sigma) W+\left(D^{2}-a^{2}\right) r-C\left(D^{2}-a^{2}\right) W \\
& \text { where } D=\frac{d}{d z}
\end{aligned}
$$


Eliminating $T$ between the equations (13) and (14), we get the single equation in $W$ in the form

$$
\left.\begin{array}{c}
{\left[\sigma+2 C \sigma^{2}-\left(D^{2}-a^{2}\right)\right]\left\{\frac{\sigma}{P_{r}}-\left(D^{2}-a^{2}\right)-\frac{Q_{s} \sigma}{P r}\left(D^{2}-a^{2}\right)\right\}\left(D^{2}-a^{2}\right) W} \\
=-R a^{2}\left[1+2 C \sigma-C\left(D^{2}-a^{2}\right)\right] W
\end{array}\right\} .
$$

The solution for $W$ for the lowest mode is taken in the form

$$
\mathrm{W}=\mathrm{A}_{0} \sin (\pi \mathrm{z})
$$

where $A_{0}$ is a constant. Substituting equation (16) in equation (15), we get

$$
\left(\sigma+2 \mathrm{C} \sigma^{2}+\mathrm{k}^{2}\right)\left\{\frac{\sigma}{\mathrm{Pr}}+\mathrm{k}^{2}+\frac{\mathrm{Q}_{s} \sigma \mathrm{K}^{2}}{\mathrm{Pr}}\right\} \mathrm{k}^{2}=\mathrm{Ra}^{2}\left(1+2 \mathrm{C} \sigma+\mathrm{Ck}^{2}\right),
$$

where $\mathrm{k}^{2}=\pi^{2}+\mathrm{a}^{2}$

Substituting $\sigma=i \omega, \omega \in \mathfrak{R}$, in equation (17) and equating the real and imaginary parts, we get the following equations

$$
\begin{aligned}
& R=\frac{T_{10}\left[k^{2} T_{11}-\omega^{2} T_{12}\right] k^{2}+2 C \omega^{2} k^{2}\left[k^{2}+T_{11} T_{12}\right]}{a^{2}\left[T_{10}^{2}+4 C^{2} \omega^{2}\right]} \\
& \omega^{2}=\frac{2 C k^{4}-T_{10} k^{2}\left[1+T_{12}\right]}{4 C^{2} k^{2}-2 C^{2} k^{2} T_{12}}
\end{aligned}
$$

where 
$\mathrm{T}_{10}=1+\mathrm{Ck}^{2}$,

$\mathrm{T}_{11}=\mathrm{k}^{2}-2 \mathrm{C} \omega^{2}$

and

$\mathrm{T}_{12}=\frac{1+\mathrm{Q}_{\mathrm{s}} \mathrm{k}^{2}}{\operatorname{Pr}}$

\section{Results and Discussion}

In this paper, we study the Rayleigh-Benard convection in Coleman - Noll fluids by replacing the classical parabolic heat equation by a non-classical heat flux law. The results obtained in the case is represented in figures (1) and (2). From figure (1) it is clear that viscoelastic parameter Qs (second-order effect) stabilises the system. From figure (2) we see that an increase in Pr and $C$ is to destabilise the system. It is also observed that the effect of Prandtl number on Rc is insensitive, this is due to the high viscosity of second-order fluids. From calculation we find that $C$ does not affect cell size at the onset of convection. We also note here that the principle of exchange of stabilities is valid in this problem if we take the parabolic heat equation and is not valid with hyperbolic heat equation.

$$
\operatorname{Pr}=10
$$

$1-a_{5}=-0.5,2-a_{5}=-0.3,3-a_{5}=0.9,4-a_{5}=0.1,5-a_{5}=0.3$

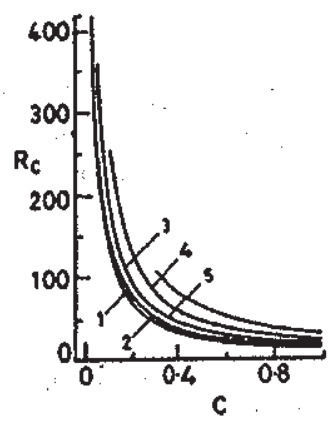

(a)

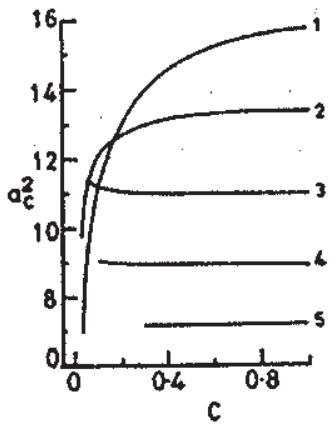

(b)

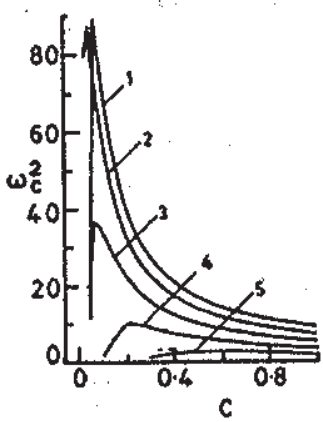

(c)

FIG. 1 PLOT OF (a) $R_{c}$, (b) $a_{c}^{2}$ AND (c) $\omega_{c}^{2}$ Vs. C FOR DIFFERENT VALUES OF $Q_{S}$ 


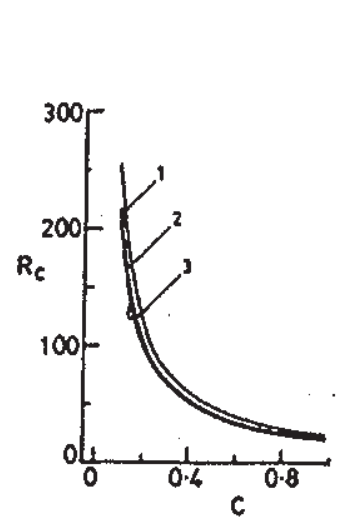

(a)
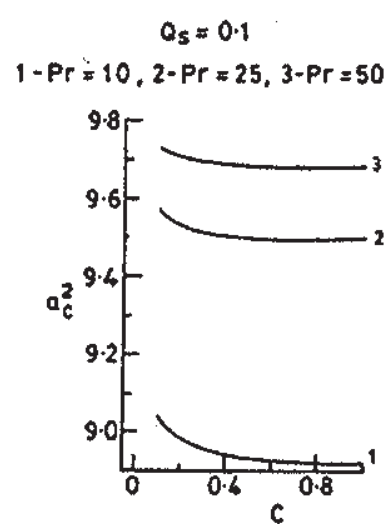

(b)

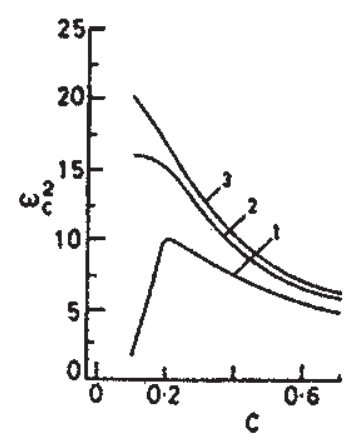

(c)

FIG. 2 PLOT OF (a) $R_{c}$, (b) $a_{c}^{2}$ AND (c) $w_{c}^{2}$ VS. C FOR DIFFERENT VALUES OF Pr

\section{Conclusion}

The non-classical Maxwell-Cattaneo heat flux law involves a wave type heat transport and does not suffer from the physically unacceptable drawback of infinite heat propagation speed. The classical Fourier flux law overpredicts the critical Rayleigh number compared to that predicted by the non-classical law. Overstability is the preferred.mode of convection.

\section{Acknowledgment}

The author would like to thank Dr. Pradeep G. Siddheshwar for suggesting the problem and for many stimulating discussions, and to the management and research development cell of Christ University for their support in completing this work.

\section{References}

1. Coleman B. D., Noll. W: An approximation theorem for functionals, with applications in continuum mechanics, Arch. Rafional Mech. Anal. Vol. 6, 355 (1960).

2. Kaloni P. N.: Some remarks on useful theorems for second-order fluids. J. Non. Newt. Fluid Mech., Vol. 31,115 (1989).

3. Sharma R. C., Pradeep Kumar: Effects of suspended particles on thermal instability in Revilin-Erickson elastico-viscous fluid, Indian J. Pure and Appl. Math., Vol. 30(5), 477 (1998). 
4. Siddheshwar P. G., Srikrishna C.V.: Weakly non-linear analysis of convection in a secondorder fluid.

5. Lindsay K. A., Straughan B: Acceleration waves and second sound in a perfect fluid. Arch. Rational Mech. Anal. Vol. 68, 53 (1978).

6. Straughan B, Franchi F:: Benard convectionand the Cattaneo law of heat conduction. Proc. Of Roy. Soc. Of Edi. 96, 175 (1984).

7. Lebon G., Cloot A.: Benard-Marangoni instability in Maxweil-Cattaneo fluid, phy. Let. 105A 361 (1984).

8. Siddheshwar P. G.,: Rayleigh-Benard convection in a magnetic field with second sound. Proc. Japan Soc. Mag. Fluids. 32, (1993).

9. Siddheshwar P. G.,Rayleigh-Benard convection in a second order ferromagnetic fluid with second sound. Proc.VIII Asian cong., Fluid Mec h. china 631, (1999).

10. Pranesh S: Effect of second sound on the onset of Rayleigh. Benard convection in a micropolar fluid, MAPANA, 12, 26, (2008).

11. Siddheshwar P. G, Pranesh S: Effect of second sound and temperature modulation on the onset convection in a Boussinesq - Stokes suspensions, (Submitted), (2008). 